\title{
GenoType MTBDRs/ performance on clinical samples with diverse genetic background
}

\author{
Paolo Miotto*, Andrea M. Cabibbe*, Paola Mantegani*, Emanuele Borroni*, \\ Lanfranco Fattorini ${ }^{\#}$, Enrico Tortoli*, Giovanni B. Migliori ${ }^{\star}$ and Daniela M. Cirillo*
}

ABSTRACT: We evaluate the performance of the GenoType ${ }_{\circledR}$ MTBDRsI (Hain Lifescience Nehren, Germany) for the detection of second-line resistant tuberculosis and we correlate the frequency of mutations to different Mycobacterium tuberculosis genotypes.

We tested 175 strains and 59 clinical specimens interpreting the results according to the Standards for Reporting of Diagnostic Accuracy recommendations. All the strains were also investigated by spoligotyping and Mycobacterial Interspersed Repetitive Units-Variable Number of Tandem Repeats typing.

The performances of the MTBDRs/ in detecting resistance to fluoroquinolones (FQ), second-line injectable drugs (SLID), and ethambutol (EMB) on clinical isolates were similar (specificity $\sim 99 \%$, sensitivity $\sim 70 \%$, and positive predictive value (PPV) $\sim 99 \%$ ).

Of the 59 respiratory specimens, three samples were classified as "indeterminate". The specificity in detecting resistances was similar for FQs and EMB 100\% (95\% Cl 92.7-100\%) and $100 \%(95 \% \mathrm{Cl} 83.9-100 \%)$, respectively with a PPV of $100 \%(95 \% \mathrm{Cl} 64.6-100 \%)$ and $100 \%(95 \% \mathrm{Cl}$ 87.9-100\%), respectively. Detection of SLID showed a specificity of $89.1 \%$ (95\% Cl $\mathbf{7 7 . 0 - 9 5 . 3 \% )}$ and a PPV of 58.3\% (95\% Cl 32.0-80.7\%). Sensitivity for FQ-resistance detection was $100 \%$ (95\% Cl $64.6-100 \%)$, whereas for SLID and EMB it was $89.1 \%(95 \% \mathrm{Cl} 77.0-95.3 \%)$ and $86.1 \%(95 \% \mathrm{CI}$ 71.3-93.9\%), respectively. We detected a significant association between mutations in the rrs gene and Beijing lineage.

The MTBDRsI can be used to "rule in" extensively drug-resistant strains of tuberculosis in a high risk group; the low sensitivity and negative predicted value (NPV) make confirmation by conventional drug susceptibility testing mandatory when mutations are not identified. NPV for SLID is higher in Beijing strains, showing that the predictive values of the molecular tests are related to the genetic background.

KEYWORDS: Extensively drug-resistant tuberculosis, GenoType ${ }^{\circledR}$ MTBDRs/, molecular drugsusceptibility testing, multidrug-resistance tuberculosis, rapid diagnosis, tuberculosis

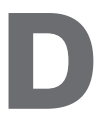
rug-resistant tuberculosis (TB) remains a major public health concern worldwide [1]. The efficacy of short-course chemotherapy is compromised by the emergence of multidrug-resistant (MDR) Mycobacterium tuberculosis strains, i.e. strains resistant to at least rifampicin and isoniazid, and of extensively drug-resistant (XDR) strains, namely MDR strains with additional resistance to any fluoroquinolone (FQ) and at least one of three second-line injectable drugs (SLIDs): capreomycin, kanamycin, amikacin [2,3]. Up to $28.3 \%$ and $61.6 \%$ of MDR-TB cases were reported among new and previously treated TB cases, respectively, and by 2010 at least one case of XDR-TB was reported in 68 countries [1].

Rapid detection of MDR-TB and XDR-TB is necessary in order to refer patients to specialist centres, thereby ensuring correct treatment under adequate infection control conditions $[4,5]$.

Currently, the direct detection of gene mutations associated with drug resistance is the only rapid method for prediction of resistance. The line probe assays (LiPA), strip-based DNA-probe tests relying

\section{AFFILIATIONS}

*Emerging Bacterial Pathogens Unit, Div. of Immunology, Transplantation and Infectious Diseases, San Raffaele Scientific Institute, Milan,

\#Dept of Infectious, Parasitic and Immune-mediated Diseases, Istituto Superiore di Sanità, Rome, and -WHO Collaborating Centre for Tuberculosis and Lung Disease, S. Maugeri Foundation, IRCCS, Tradate, Italy.

\section{CORRESPONDENCE}

D.M. Cirillo

Emerging Bacterial Pathogens Unit Div. of Immunology, Transplantation and Infectious Diseases San Raffaele Scientific Institute Via Olgettina 58 20132 Milano Italy

E-mail: cirillo.daniela@hsr.it

Received:

Sept 202011

Accepted after revision:

Dec 232011

First published online:

Jan 202012 
on multiplex nucleic acid amplification and reverse hybridisation, are commercially available for the detection of multiple antibiotic resistances.

In particular, the GenoType MTBDR and MTBDRplus assays (both Hain Lifescience, Nehren, Germany) showed to be highly sensitive and specific assays for the prediction of rifampicin and, at a lower extent, of isoniazid resistances on sputum smearpositive samples [6].

In 2008, the World Health Organization issued (WHO) a policy statement for rapid screening of patients at risk of MDR-TB, providing recommendations on the use of molecular LiPA in TB national programmes $[6,7]$. Their use greatly reduced the time in which MDR-TB cases were detected in high-risk populations, thereby preventing the development of resistance due to patient mismanagement.

Recently, a second generation LiPA MTBDRsl was developed to detect the most frequent mutations responsible for resistance to the XDR-TB defining drugs, FQ and SLIDs, plus ethambutol (EMB). Few studies compared the MTBDRsl to genetic sequencing of clinical isolates and assessed its performance by comparison with the gold standard, the phenotypic drugsusceptibility testing (DST) [8-12]; furthermore, only one such study evaluated it directly on clinical specimens [8]. More data are, therefore, needed to better understand the usefulness of this assay in the clinical practice.

So far, no study reported genotyping information concerning strains analysed. Association between genotypes and mutation frequency in genes involved in resistance was only accomplished for first-line drug (FLD) resistance and no data are available for genes involved in second-line drug (SLD) resistance [13, 14]. Here, we evaluated the performance of the MTBDRsl by comparison with DST and sequencing on 175 clinical isolates and 59 clinical specimens. Furthermore, for the first time, we reported associations between mutations involved in resistances to SLIDs and different $M$. tuberculosis genotypes. We provided the proof-of-principle on how the performances of a molecular test can be altered in different geographical settings by considering the phylogeographical distribution of M. tuberculosis.

\section{MATERIALS AND METHODS Clinical isolates}

175 M. tuberculosis cultures, with known phenotypic resistant patterns, were studied by the MTBDRsl assay (table 1).

\begin{tabular}{|c|c|c|c|c|}
\hline TAB & $\begin{array}{l}\text { Phe } \\
\text { clin } \\
\text { incl }\end{array}$ & $\begin{array}{l}\text { drug } \\
\text { tes } \\
\text { the }\end{array}$ & 10 & $\begin{array}{l}\text { f the } \\
\text { ns }\end{array}$ \\
\hline \multirow[t]{2}{*}{ Drug } & \multicolumn{2}{|c|}{ Clinical isolates } & \multicolumn{2}{|c|}{ Clinical specimens } \\
\hline & $\mathbf{R}$ & s & $\mathbf{R}$ & s \\
\hline FQs & 57 & 118 & 7 & 52 \\
\hline SLIDs & 85 & 90 & 10 & 49 \\
\hline EMB & 84 & 91 & 17 & 29 \\
\hline
\end{tabular}

FQs: fluoroquinolones; SLIDs: second-line injectable drugs; EMB: ethambutol R: resistant; S: susceptible.

\section{Clinical specimens}

DNA obtained from 59 clinical specimens from MDR cases that had been decontaminated, according to international guidelines using $\mathrm{N}$-acetyl-cysteine/ $\mathrm{NaOH}$ procedure, were included in the study. All samples were acid-fast bacilli-positive, according to sputum smear microscopy. The susceptibility phenotypes of the corresponding strains had been determined.

\section{DST}

Susceptibility to FLD and SLD was performed by the BACTEC ${ }^{\mathrm{TM}}$ MGIT $^{\mathrm{TM}} 960$ system (MGIT) (BD Bioscience, Erebodegem, Belgium) [15].

\section{DNA extraction}

Genomic DNA was extracted from strains and clinical specimens as described elsewhere [16].

\section{GenoType $_{\circledR}$ MTBDRsl assay}

Amplification of DNA extracted from isolates was performed accordingly to the manufacturer's instruction. For the sputum specimens, a modified amplification protocol was applied: 40 cycles instead of 20 and $2 \mathrm{U}$ HotStarTaq DNA polymerase were used.

\section{Sequencing}

Genomic regions harbouring drug-resistance mutations were amplified and sequenced using primers specific for each genomic region (table S1).

\section{Spoligotyping}

The amplified direct repeat locus of clinical isolates was investigated using the spoligotyping kit (Ocimum Biosolutions, Hyderabad, India) following the manufacturer's instructions. Spoligotyping results were entered, in binary format, in MIRUVNTRplus web database to identify spoligotype international types (SITs) and strain main lineages [17]. Best-matching lineages were identified by similarity search (distance setting: $<0.1$ ).

\section{Microplate Alamar blue assay}

The assay was performed according to standard procedure with minor modifications [18]. EMB concentrations ranged from $256 \mu \mathrm{g} \cdot \mathrm{mL}^{-1}$ to $1 \mu \mathrm{g} \cdot \mathrm{mL}^{-1}$. M. tuberculosis $\mathrm{H} 37 \mathrm{Rv}$ was used as a drug-susceptible reference strain. Strains showing a minimum inhibitory concentration (MIC) $\geqslant 4 \mu \mathrm{g} \cdot \mathrm{mL}^{-1}$ were considered EMB resistant.

\section{Statistical analysis}

To evaluate the MTBDRsl, we compared the LiPA performance to DST performed by MGIT and to direct sequencing as reference standards following the Standards for Reporting of Diagnostic Accuracy (STARD) recommendations [19]. Mid-p exact test was applied to assess the significance of different mutations frequencies among different genotypes. Sensitivity, specificity, positive predictive value (PPV), negative predictive value (NPV), likelihood ratios and diagnostic accuracy values were calculated according to the Wilson score. All statistical analyses were carried out using the Open Source Epidemiologic Statistics for Public Health version 2.3.1 [20]. 


\begin{tabular}{|c|c|c|c|c|}
\hline TABLE 2 & $\begin{array}{l}\text { toroquinolo } \\
\text { eType } \\
5 \text { clinical is }\end{array}$ & $\begin{array}{l}\text { le resistance } \\
\text { TBDRs/ assa } \\
\text { lates and } 59\end{array}$ & $\begin{array}{l}\text { results obt } \\
\text { ay and sequ } \\
9 \text { clinical sp }\end{array}$ & $\begin{array}{l}\text { ained by } \\
\text { encing on } \\
\text { ecimens }\end{array}$ \\
\hline M. tuberculosis & $\begin{array}{c}\text { Phenotypic } \\
\text { DST }\end{array}$ & MTBDRsI & Sequencing & n \% \\
\hline \multicolumn{5}{|l|}{ Clinical isolate } \\
\hline & $\mathrm{R}$ & A90V & A90V & $15(8.6)$ \\
\hline & R & D94G & D94G & $11(6.3)$ \\
\hline & $\mathrm{R}$ & S91P & S91P & $4(2.3)$ \\
\hline & R & D94A & D94A & $2(1.1)$ \\
\hline & R & D94N/D94Y & D94N & $2(1.1)$ \\
\hline & $\mathrm{R}$ & no WT & D94Y & $2(1.1)$ \\
\hline & $\mathrm{R}$ & WT+D94A & WT+D94A & $1(0.6)$ \\
\hline & R & WT+D94A & WT & $1(0.6)$ \\
\hline & R & WT+D94G & WT & $2(1.1)$ \\
\hline & $\mathrm{R}$ & WT+D94G & D94G & $1(0.6)$ \\
\hline & $\mathrm{R}$ & D94A+D94G & D94A & $1(0.6)$ \\
\hline & $\mathrm{R}$ & WT & WT & $15(8.6)$ \\
\hline & S & WT & WT & $116(66.3)$ \\
\hline & S & No WT & WT & $1(0.6)$ \\
\hline & S & Indeterminate & WT & $1(0.6)$ \\
\hline \multicolumn{5}{|l|}{ Clinical specimen } \\
\hline & R & A90V & & $1(1.7)$ \\
\hline & R & D94G & & $2(3.4)$ \\
\hline & $R$ & D94N/D94Y & & $3(5.1)$ \\
\hline & R & No WT & & $1(1.7)$ \\
\hline & S & WT & & $49(83.1)$ \\
\hline & S & Indeterminate & & $3(5.1)$ \\
\hline
\end{tabular}

DST: drug-susceptibility testing; R: resistant; WT: wild type; S: susceptible.

\section{RESULTS}

\section{Performance of MTBDRsI on clinical isolates}

The performance of the MTBDRsl assay was evaluated on 175 clinical isolates. An indeterminate result was obtained in one isolate due to lack of hybridisation.

\section{FQs}

The panel strains included 57 phenotypically resistant isolates and 117 FQ-susceptible strains (table 2). FQ-resistant strains were correctly identified by MTBDRsl in $73.7 \%$ of cases. In six cases both wild-type (WT) and mutated DNA sequences were detected, a finding suggestive of a mixed infection. In two cases a mutation in the gyrA gene was inferred by the lack of hybridisation of the wild-type probe WT3; however, the aminoacid substitution D94Y was not detected by the specific probe. The codons 94 and 90 were the most frequently affected by mutations $(26.3 \%$ and $19.3 \%$ of resistant strains, respectively). The mutation S91P was detected in $7.0 \%$ cases. $26.3 \%$ of strains, phenotypically resistant to $F Q$, were classified as susceptible because of hybridisation of all the WT gyrA probes (data confirmed by sequencing).

All but one sample of the phenotypically susceptible strains were correctly recognised by the test. Diagnostic performances are summarised in table 3. Comparison between GenoType ${ }^{\circledR}$
MTBDRsl and sequencing data analysis are reported in the online supplementary data (table S2).

Genotype analysis of clinical isolates with known FQ susceptibility was investigated (table S3). Among them, 76.9\% of the FQ-resistant strains were affected by gyrA mutations (tables 4 , S4). Within non-Beijing lineages, the proportion of gyr $A$ mutated and non-mutated FQ-resistant strains was grossly overlapping; no significant differences were observed between Beijing and non-Beijing lineages ( $p>0.05)$.

\section{SLIDS}

Out of the 174 strains evaluated, 84 were resistant to at least one SLID and 90 were susceptible to all drugs (table 1). 60 resistant strains $(71.4 \%)$ were detected by the MTBDRsl assay (table 5), among which five showed a double pattern. In one, a mutation in a subregion of the rrs gene was inferred by lack of hybridisation with the probe WT1 (nucleotides 1401-1402). One strain showing hybridisation with the probe MUT2 (specific for the mutation g1484t) turned out to be WT by sequencing. The substitution a1401g was the most frequent affecting 57 (67.9\%) out of the 84 resistant strains (table S5). The substitution c1402t was observed only once $(1.2 \%)$. 24 strains, which were phenotypically resistant to SLID, were classified as susceptible; the WT sequence in rrs was confirmed by sequencing.

All strains phenotypically susceptible to the three SLIDs were correctly recognised by the test, diagnostic performances are summarised in table 3. Comparison of MTBDRsl and sequencing data analysis are reported online in the supplementary data (table S2).

Among the strains with known phenotypic results to all three SLIDs, the a1401g substitution correlated with phenotypic resistance to at least two drugs among kanamycin, capreomycin, amikacin (57 (87.7\%) out of 65 cases).

The Beijing spoligotype was the most prevalent (table S3; mutation details are given in table S6). Among SLID-resistant strains the $r r s$ mutations were present in $78.3 \%$ of the Beijing lineage and in only $59.0 \%$ of the non-Beijing lineage (table 4 ). The difference was statistically significant $(\mathrm{p}<0.01)$.

\section{$E M B$}

83 phenotypically EMB-resistant and 91 EMB-susceptible strains were tested; table 6 shows the comparison between LiPA and sequencing.

Among 83 phenotypically resistant strains, 55.4\% were correctly identified by MTBDRsl. In three cases the mutation inferred by the lack of hybridisation with the WT probe was confirmed by genetic sequencing that detected substitutions corresponding to M306I (atg/atc) and M306L respectively. The most frequent mutations were M306V and M306I observed in $42.2 \%$, and $12.0 \%$ of phenotypically resistant strains, respectively. $37(44.6 \%)$ strains phenotypically resistant to EMB were classified as susceptible (hybridisation with the WT probe, confirmed by sequencing). Table S7 shows embB differences.

Phenotypical susceptibility to EMB was correctly recognised in $54.9 \%$ of strains. In $28.6 \%$ of the 41 discrepant cases both MTBDRsl and direct sequencing identified a specific mutation at codon 306. 12 additional strains identified by MTBDRsl as resistant, by the lack of WT hybridisation, harboured the 
TABLE 3 Statistical parameters of the performances of the GenoType ${ }_{\mathbb{B}}$ MTBDRs/ assay on 175 clinical isolates and 59 clinica specimens

\begin{tabular}{|c|c|c|c|c|}
\hline \multirow[t]{2}{*}{ Drug } & \multicolumn{2}{|c|}{ Clinical isolates } & \multicolumn{2}{|c|}{ Clinical specimens } \\
\hline & MTBDRsI & DST & MTBDRsI & DST \\
\hline \multicolumn{5}{|l|}{ Fluoroquinolones } \\
\hline Sensitivity & $99.4(96.8-99.9)$ & $73.7(61.0-83.4)$ & $94.9(86.1-98.3)$ & $100(64.6-100)$ \\
\hline Specificity & & $99.2(95.3-99.9)$ & & $100(92.7-100)$ \\
\hline PPV & & $97.7(87.9-99.6)$ & & $100(64.6-100)$ \\
\hline NPV & & $88.6(82.0-92.9)$ & & $100(92.7-100)$ \\
\hline Likelihood ratio pos. & & $86.2(11.9-62.2)$ & & Undefined \\
\hline Likelihood ratio neg. & & $0.3(0.2-0.3)$ & & 0 \\
\hline Diagnostic accuracy & & $90.8(85.6-94.3)$ & & $100(93.6-100)$ \\
\hline \multicolumn{5}{|c|}{ Second-line injectable drugs } \\
\hline Sensitivity & $99.4(96.8-99.9)$ & $71.4(61.2-80.0)$ & $91.5(81.7-96.3)$ & $87.5(52.9-97.8)$ \\
\hline Specificity & & $100(95.9-100)$ & & $89.1(77.0-95.3)$ \\
\hline PPV & & $100(94.0-100)$ & & $58.3(32.0-80.7)$ \\
\hline NPV & & $79.0(70.6-85.4)$ & & $97.6(87.7-99.6)$ \\
\hline Likelihood ratio pos. & & Undefined & & $8.1(5.2-12.4)$ \\
\hline Likelihood ratio neg. & & $0.3(0.3-0.3)$ & & $0.1(0.02-1.0)$ \\
\hline Diagnostic accuracy & & $86.2(80.3-90.6)$ & & $88.8(77.8-94.8)$ \\
\hline \multicolumn{5}{|l|}{ Ethambutol $^{\#}$} \\
\hline Sensitivity & $99.4(96.8-99.9)$ & $69.7(61.0-77.1)$ & $94.9(86.1-98.3)$ & $86.1(71.3-93.9)$ \\
\hline Specificity & & $96.2(87.0-98.9)$ & & $100(83.9-100)$ \\
\hline PPV & & $97.7(92.0-99.4)$ & & $100(87.9-100)$ \\
\hline NPV & & $57.5(47.0-67.3)$ & & $80.0(60.9-91.1)$ \\
\hline Likelihood ratio pos. & & $18.1(6.7-47.8)$ & & Undefined \\
\hline Likelihood ratio neg. & & $0.3(0.3-0.3)$ & & $0.1(0.09-0.2)$ \\
\hline Diagnostic accuracy & & $77.6(70.8-83.2)$ & & $91.1(80.7-96.1)$ \\
\hline
\end{tabular}

Data are presented as \% (95\% Cl \%). DST: drug-susceptibility testing; interval; PPV: positive predictive value; NPV: negative predictive value; pos: positive; neg: negative; inject: injectable. ${ }^{\#}$ : the phenotypic DST result is adjusted according to the minimum inhibitory concentration results.

TABLE 4 Genotype analysis of clinical Mycobacterium tuberculosis isolates with known drugsusceptibility testing and mid-p exact test on the distribution of mutations among lineages

\begin{tabular}{|c|c|c|c|}
\hline \multirow[t]{2}{*}{ Drug } & \multicolumn{2}{|c|}{ M. tuberculosis strain } & \multirow[t]{2}{*}{ p-value } \\
\hline & Beijing & Other ${ }^{\#}$ & \\
\hline \multicolumn{4}{|c|}{ Fluoroquinolones } \\
\hline \multicolumn{4}{|l|}{ gyrA } \\
\hline Wild-type & 23.1 & 29.0 & 0.166 \\
\hline Mutated & 76.9 & 71.0 & \\
\hline \multicolumn{4}{|c|}{ Second-line injectable drugs } \\
\hline \multicolumn{4}{|l|}{ rrs } \\
\hline Wild-type & 21.7 & 41.0 & $0.002^{\bullet}$ \\
\hline Mutated & 78.3 & 59.0 & \\
\hline \multicolumn{4}{|l|}{$\begin{array}{c}\text { Ethambutol } \\
e m b B\end{array}$} \\
\hline Wild-type & 29.5 & 30.0 & 0.470 \\
\hline Mutated & 70.5 & 70.0 & \\
\hline
\end{tabular}

Data presented as \% unless otherwise stated. ${ }^{\#}$ : all other lineages excluding Beijing.": statistically significant.
M306I mutation (codon substitution occurred in atg306atc $(\mathrm{n}=8)$ and atg306att $(\mathrm{n}=4))$. In two cases the MTBDRsl showed a double pattern; sequencing resulted WT in the first case and detected only the M306I mutation in the second case. Finally, in the last discrepant case, a faint hybridisation signal lead to misinterpretation but, after re-checking, the presence of M306I substitution was acknowledged.

The MICs of 33 drug-susceptible isolates harbouring embB mutations (table 7) confirmed a resistant phenotype in 31 cases. Diagnostic performances are summarised in table 3. Comparison between MTBDRsl and sequencing data analysis are reported in table S2.

The spoligotype of 175 strains in which EMB resistance was investigated is reported in table S3 (phenotypic DST result is adjusted according to the MIC results). The proportion of mutated and non-mutated EMB-resistant strains within Beijing and nonBeijing lineages (table 4 and table S8) was similar ( $>00.05)$.

Overall performances of the MTBDRsl on different strain lineages are reported in table S9.

\section{Performance of MTBDRsI on clinical specimens}

Of the 59 respiratory specimens tested with MTBDRsl 53 showed valid results. Three samples $(5.1 \%)$ did not provide 


\begin{tabular}{|c|c|c|c|c|}
\hline \multicolumn{5}{|l|}{ Clinical isolate } \\
\hline & $\mathrm{R}$ & a1401g & a1401g & 53(30.3) \\
\hline & R & WT & WT & $24(13.7)$ \\
\hline & $\mathrm{R}$ & $a 1401 g+g 1484 t$ & a1401g & $3(1.7)$ \\
\hline & $\mathrm{R}$ & WT+a1401g & WT & $1(0.6)$ \\
\hline & $\mathrm{R}$ & $g 1484 t$ & WT & $1(0.6)$ \\
\hline & $\mathrm{R}$ & Indeterminate & WT & $1(0.6)$ \\
\hline & $\mathrm{R}$ & WT & & $1(1.7)$ \\
\hline & $\mathrm{R}$ & Indeterminate & & $2(3.4)$ \\
\hline & S & WT & & $41(69.5)$ \\
\hline & S & $W T+g 1484 t$ & & $4(6.8)$ \\
\hline & S & No WT (c1402t) & & $1(1.7)$ \\
\hline & S & Indeterminate & & $3(5.1)$ \\
\hline
\end{tabular}

DST: drug-susceptibility testing; R: resistant; WT: wild type; S: susceptible.

valid hybridisation for any of the three genomic regions and were classified as "indeterminate". The overall diagnostic performances are summarised in table 3 .

\section{$F Q$}

Table 2 shows the comparison between LiPA results and the DST performed on the corresponding strain. The MTBDRsl identified seven resistant strains, all confirmed by phenotypic DST; the remaining 49 samples, characterised by a WT pattern, were FQ-susceptible by DST. Overall concordance was $94.9 \%$.

\section{SLIDS}

Table 5 shows the comparison between LiPA results and the DST performed on the corresponding strain. Twelve samples were classified resistant by MTBDRsl; in four a mutated pattern (a1401g) was detected, in one hybridisation of the WT probe was lacking and in seven a double pattern was present. Seven samples showing positive hybridisation with the a1401 $\mathrm{g}$ probe were confirmed to be resistant by phenotypic DST. On the contrary specimens lacking hybridisation with the WT probe or hybridising with the a1484t probe were phenotypically susceptible to all three SLIDs. From the 42 samples showing a WT hybridisation, 41 were phenotypically susceptible while one was amikacin-resistant. Two samples, phenotypically resistant to kanamycin, failed hybridisation with the rrs probe and were considered indeterminate. The concordance between the MTBDRsl assay and phenotypic DST was $81.4 \%$.

\section{$E M B$}

The MTBDRsl identified EMB resistance in 31 samples by detection of substitutions and lack of hybridisation with the WT probe (table 6). Only 12 cases were confirmed resistant by phenotypic DST. From the 25 specimens showing a WT hybridisation pattern, 20 were confirmed as susceptible by DST whereas five were found to be resistant. Overall concordance was $55.9 \%$.

\section{DISCUSSION}

The concordance between MTBDRsl and sequencing was $>95 \%$ for all genomic regions analysed. Discrepancies were mainly due to double patterns (WT+mutation) whose problematic detection by sequencing is known. Among clinical isolates, $\mathrm{WT}+$ mutation patterns were rarely found (six cases for gyrA gene, five for $r$ rs gene and two for $e m b B$ gene) and exclusively in phenotypically resistant isolates. Only in one case the MTBDRsl reported a mutation (a1484t, rrs gene) that could not be detected by sequencing. The high agreement between MTBDRsl and sequencing is in line with previous observations [8-12].

While on clinical isolates the molecular assay was repeated in three cases to obtain a valid result $(1.7 \%)$, on clinical specimens the molecular assay was repeated in nine cases $(16.1 \%)$. The majority of anomalies were related to the rrs amplification (e.g. faint band staining, no amplification). In some cases sample freezing and thawing may have decreased the PCR performance. The wide range of confidence intervals of the performances on clinical specimens reflects the low number of resistant clinical specimens available for this study and the high frequency of double pattern (especially for SLIDs-resistance). However, prevalence of $11.9 \%$ for FQ-resistance and of $15.3 \%$ for SLID-resistance was observed. Despite the lack of clear data, prevalence for FQ-resistance or SLID-resistance among TB cases within low-burden countries is within the range of $10 \%-15 \%$. 
TABLE 6 Ethambutol resistance: comparison of results obtained by GenoType $e_{\circledast}$ MTBDRs/ assay and by sequencing on 175 clinical isolates and 59 clinical specimens

\begin{tabular}{|c|c|c|c|c|}
\hline M. tuberculosis & Phenotypic DST & MTBDRsI & Sequencing & n (\%) \\
\hline \multicolumn{5}{|l|}{ Clinical isolates } \\
\hline & $\mathrm{R}$ & M306I & M306I & $8(4.6)$ \\
\hline & $\mathrm{R}$ & No WT & ${\mathrm{M} 306 \mathrm{I}^{\#}}^{\#}$ & $2(1.1)$ \\
\hline & $\mathrm{R}$ & & M306L & $1(0.6)$ \\
\hline & S & M306V & M306V & $16(9.1)$ \\
\hline & S & M306I & M306I & $10(5.7)$ \\
\hline & S & & WT & $1(0.6)$ \\
\hline & S & No WT & M306I $^{\#}$ & $8(4.6)$ \\
\hline & S & & M3061 & $4(2.3)$ \\
\hline & S & WT+M306V & WT & $1(0.6)$ \\
\hline & $\mathrm{R}$ & M306V & & $11(18.6)$ \\
\hline & R & M306I & & $2(3.4)$ \\
\hline & $\mathrm{R}$ & WT & & $5(8.5)$ \\
\hline & $\mathrm{R}$ & Indeterminate & & $2(3.4)$ \\
\hline & S & WT & & 20 (33.9) \\
\hline & $\mathrm{s}$ & M3066 V & & $7(11.9)$ \\
\hline & S & M306I & & $9(15.3)$ \\
\hline & S & WT+M306V & & $1(1.7)$ \\
\hline & $S$ & No WT & & $1(1.7)$ \\
\hline & S & Indeterminate & & $1(1.7)$ \\
\hline
\end{tabular}

DST: drug-susceptibility testing; R: resistant; WT: wild type; S: susceptible; ${ }^{\#}$ : atg $\rightarrow$ atc; ${ }^{\bullet}$ : atg $\rightarrow$ att.

Thus, our sample size provides the evaluation of the diagnostic performances of the MTBDRsl in a real setting.

\section{FQs}

As to clinical samples and isolates, the MTBDRsl was $99 \%$ specific in detecting FQ resistance, showing a PPVs of $\sim 98 \%$. In contrast, sensitivity was significantly lower $(73.7 \%)$, probably because of mutations conferring FQ-resistance in genomic regions not targeted by MTBDRsl [21, 22]. The NPV (88.6\%) makes confirmation by conventional DST mandatory whenever a susceptible MTBDRsl pattern is obtained.

\section{SLID}

The performances of the MTBDRsl on SLIDs were similar to those of FQ (99.4\% specificity, $71.4 \%$ sensitivity and 100\% PPV). Indeed, the assay targets only one region involved in the development of resistant phenotypes $[22,23]$. Due to the low NPV value $(79.0 \%)$, confirmation by conventional DST is required for every strain presenting susceptible MTBDRsl pattern.

Five false SLIDs resistances significantly affected the MTBDRsl performance in clinical specimens. Four were characterised by double pattern compatible with a mixed infection (susceptible and a resistant strain) while the fifth, identified as resistant by lack of hybridisation with the WT probe, presented anomalies in other molecular assays, suggesting presence of inhibitors or
DNA degradation. However, a point of note is that the patient was still sputum smear-positive after 12 months, despite being treated for MDR-TB according to the WHO guidelines [4].

To our knowledge, this is the first report of hetero-resistance related to SLIDs. DST could not confirm a mixed, resistant and susceptible, population. However, it is possible that a predominant proportion of WT bacteria conceal the resistance in the phenotypic test as observed by HillemanN et al. [8].

Several groups correlated rrs mutations with cross-resistance to amikacin, kanamycin and capreomycin [23, 24]. We observed that the a1401g substitution correlated with phenotypic resistance to at least two SLID (87.5\% of cases). Once confirmed, detection of such mutation could provide a useful hint in a patient's management. Like HiLLEMANN et al. [8] we identified a strain resistant to all three SLIDs harbouring a c1402t substitution in rrs, which is in contrast with a report correlating this mutation to phenotypic resistance to capreomycin and kanamycin only $[8,24]$. Thus, a relationship between rrs mutations and SLID cross-resistances is still unknown.

\section{EMB}

MTBDRsl provided discrepant results on EMB resistance. In comparison with phenotypic DST, the MTBDRsl showed low specificity and sensitivity (55\%), mainly due to $e m b B$ mutations 


\begin{tabular}{|c|c|c|c|c|}
\hline \multirow{3}{*}{$\begin{array}{l}\text { TABLE } 7 \\
\text { ERDR } \\
\text { sequence }\end{array}$} & \multicolumn{4}{|c|}{$\begin{array}{l}\text { Correlation of mutations, detected by } \\
\text { sequencing of the ethambutol (EMB)-resistance } \\
\text { determining region, to the minimum inhibitory } \\
\text { concentrations (MICs) of } 33 \text { clinical isolates } \\
\text { phenotypically susceptible }{ }^{\#} \text { to EMB }\end{array}$} \\
\hline & \multirow{2}{*}{$\begin{array}{c}\text { Conventional } \\
\text { DST }\end{array}$} & \multicolumn{2}{|c|}{ MIC MABA } & \multirow[t]{2}{*}{$\mathbf{n}$} \\
\hline & & $\mu \mathrm{g} \cdot \mathrm{mL}^{-1}$ & Result & \\
\hline \multirow[t]{4}{*}{ M306V } & S & 32 & $\mathrm{R}$ & 1 \\
\hline & S & 16 & $\mathrm{R}$ & 5 \\
\hline & $S$ & 8 & $\mathrm{R}$ & 4 \\
\hline & S & 4 & $\mathrm{R}$ & 1 \\
\hline \multirow[t]{2}{*}{ M306I } & S & 8 & $\mathrm{R}$ & 5 \\
\hline & S & 4 & $\mathrm{R}$ & 4 \\
\hline \multirow[t]{4}{*}{ M306 $1^{\circ}$} & S & 16 & $R$ & 2 \\
\hline & S & 8 & $\mathrm{R}$ & 2 \\
\hline & S & 4 & $\mathrm{R}$ & 2 \\
\hline & S & 2 & S & 1 \\
\hline \multirow[t]{2}{*}{${\mathrm{M} 306 \mathrm{I}^{+}}^{+}$} & S & 8 & $R$ & 1 \\
\hline & S & 2 & S & 1 \\
\hline \multirow[t]{2}{*}{ S297A } & $S$ & 16 & $\mathrm{R}$ & 1 \\
\hline & S & 4 & $\mathrm{R}$ & 1 \\
\hline S296H & S & 8 & $R$ & 1 \\
\hline S347I & S & 8 & $\mathrm{R}$ & 1 \\
\hline
\end{tabular}

ERDR: EMB-resistance determining region; DST: drug-susceptibility testing; MABA: microplate Alamar blue assay; S: susceptible; R: resistant; \#: BACTEC $^{\text {TM }}$ MGIT $^{\text {TM }} 960$ system was used for detection; " : atg $\rightarrow$ atc; ${ }^{+}:$atg $\rightarrow$ att.

at codon 306 in 41 phenotypically susceptible strains. Similar results were obtained in clinical specimens.

MIC values resolved the discrepancies between LiPA and DST but raised concerns on phenotypic EMB DST by MGIT. Our results support the findings of PLINKE et al. [25] reporting that strains, phenotypically susceptible to EMB and carrying mutations at codon 306 of the $e m b B$ gene, were found resistant after retesting. These findings raise concerns regarding the standardisation of phenotypic DST for EMB. In fact, recent results on the proficiency testing of DST in supranational TB reference laboratories reported by VAN DEUN et al. [26] highlighted a lack of consistency in DST results for EMB. In particular, sensitivity was reported to be $\sim 93 \%$, the worst performance among all the first line drugs tested (rifampin, isoniazid and streptomycin). EMB testing was also the DST showing the higher number of rounds with sensitivity $<80 \%$ and the lower with sensitivity $>100 \%$. In addition, also the reproducibility was found to be poor [26]. Despite the lack of clear experimental evidences, we could hypothesise a role in resistance determination for the medium used for the test; thus the cut-off value selected to perform DST for EMB by MGIT $\left(5 \mu \mathrm{g} \cdot \mathrm{mL}^{-1}\right)$ might underestimate resistance. Indeed, SCARPARO et al. [27] showed that the MGIT system provided very major errors (false-susceptible results) only for EMB using as a cut-off value $5 \mu \mathrm{g} \cdot \mathrm{mL}^{-1}$. Thus, when MIC results were used for assessment of EMB tests, MTBDRsl specificity and sensitivity were raised up to $96.2 \%$ and $69.7 \%$, respectively, and the PPV increased from $56.2 \%$ to $97.7 \%$ in clinical isolates.
Low NPV values $(57.5 \%)$ are likely to be caused by the presence on the MTBDRsl of only the codon 306 of embB.

\section{Genetic diversity and frequency of mutations}

Previous studies, demonstrated that the population genetics of M. tuberculosis is highly geographically structured [13, 28]: each of the main lineages is associated with particular geographical areas. In fact, a high correlation between genetic variability and geographical areas exists, and has been estimated taking into account human demographics and migration [28]. Thus, for each geographical setting it might be possible to identify a prevalent $M$. tuberculosis lineage and it has been hypothesised that phylogeographical diversity may influence the development of new diagnostics, drugs, and vaccines [13]. Some lineages show a higher frequency of mutations on "canonical" genes conferring antibiotic resistance. It seems likely that the performances of molecular assays may, therefore, be affected by the mycobacterial lineage transmitted within a particular geographical setting.

So far association studies between mycobacterial genotypes and incidence of mutations in drug-resistance genes are available only for rifampicin and isoniazid and are missing for SLID resistance genes [14]. The frequency of mutations within the major lineages identified by spoligotyping enables us to detect a statistically relevant association between rrs mutations and Beijing strains. In contrast, no association was found for EMBrelated or FQ-related mutations.

To avoid inclusion of clonal strains in our analysis, we performed the Mycobacterial Interspersed Repetitive Units-Variable Number of Tandem Repeats (MIRU-VNTR) genotyping technique on all isolates belonging to the Beijing lineage. Once phenotypic DST, year and country of isolation, and single nucleotide polymorphisms within drug-resistance genes $(g y r A, r r s, e m b B$, rpo $B$, inh $A$, $k a t G$, gidB, tly $A$, and eis) were analysed together with the MIRUVNTR genotype (data not shown) the Beijing lineage showed a sufficient degree of diversity to exclude a clonal origin. Our data seemed, therefore, to confirm that Beijing strains may be characterised by higher mutation frequencies and that their cellwall characteristics increased MDR and hyper-virulent phenotypes [14].

\section{Clinical consequences}

Determination of phenotypic DST to FLD and SLID requires 49 weeks $[29,30]$. The use of rapid molecular assays reduces the time for diagnosing MDR-TB and XDR-TB to just 1-2 days. Can we speculate that the use of the MTBDRsl in clinical routine allows the identification of FQ-resistant and/or SLID-resistant cases in 1-2 days since the detection of MDR phenotype?

$13(46.4 \%)$ out of 28 XDR-TB strains of our panel were recognised by MTBDRsl to have both gyrA and rrs mutations. In seven cases $(25 \%)$ the assay identified FQ resistance and in four cases $(14.3 \%)$ to SLID resistance; four XDR-TB strains not harbouring mutations in the genes targeted by the MTBDRsl were misidentified as susceptible. Therefore, further investigations are needed to better understand the clinical add-on value of molecular assay in detecting XDR-TB cases correctly.

Due to the high PPV and specificity, the MTBDRsl allows detection of phenotypically resistant cases; anyway, low sensitivity and NPV make confirmation by conventional DST mandatory on all cases classified as susceptible by the molecular test. The study 
results confirm that the field of application of MTBDRsl is the screening of selected MDR-TB patients at higher risk of XDR-TB (e.g. contacts of XDR-TB cases, treatment failures, screening of the index case contacts in specific outbreaks, etc.). Addition of further genomic targets involved in resistant phenotype to FQ and SLID could improve performances of the MTBDRsl in the next future.

Important advantages of the MTBDRsl test are the ability of identifying both of hetero-resistance and mutations conferring low-level resistance. Hetero-resistance is in fact the background for development of resistances and low-level resistance before high-level resistance [31].

To our current knowledge this study is the first to investigate the distribution of SLID-related mutations among $M$. tuberculosis lineages. It seems plausible that the MTBDRsl performs differently in different geographical settings. This finding is relevant in clinical practice, thus the contribution of molecular tests aiming at identifying drug resistances should be evaluated considering local epidemiological features.

In conclusion, our study strengthens previous findings concerning the performances of the MTBDRsl on a larger set of samples. In addition, we provided a correlation between strain lineages and frequency of mutations occurring in SLID-resistant strains, supporting the hypothesis that performances of molecular tests can be affected by the phylogeographical features.

\section{STATEMENT OF INTEREST}

A statement of interest for this study can be found at www.erj. ersjournals.com/site/misc/statements.xhtml

\section{ACKNOWLEDGEMENTS}

This research received funding from the European Community's Seventh Framework Programme (FP7/2007-2013) under grant agreements FP7-202145 (TM-REST) and FP7-223681 (TB-PANNET) to DMC. We would like to thank G. Sotgiu (Epidemiology and Medical Statistics Unit, Dept of Biomedical Sciences, University of Sassari, Sassari, Italy) and R. Centis (WHO Collaborating Centre for Tuberculosis and Lung Disease, S. Maugeri Foundation, IRCCS, Tradate, Italy) for useful discussions and Hain Lifescience (Nehren, Germany) for providing the GenoType ${ }^{\circledR}$ MTBDRsl assay free of charge.

\section{REFERENCES}

1 World Health Organization. Global Tuberculosis Control: WHO report 2010. WHO/HTM/TB/2010.7. Geneva, World Health Organization, 2010.

2 Yew WW, Lange C, Leung CC. Treatment of tuberculosis: update 2010. Eur Respir J 2011; 37: 441-462.

3 Sotgiu G, Ferrara G, Matteelli A, et al. Epidemiology and clinical management of XDR-TB: a systematic review by TBNET. Eur Respir J 2009; 33: 871-881.

4 Falzon D, Jaramillo E, Schünemann HJ, et al. WHO guidelines for the programmatic management of drug-resistant tuberculosis: 2011 update. Eur Respir J 2011; 38: 516-628.

5 Sotgiu G, D'Ambrosio L, Centis R, et al. TB and M/XDR-TB infection control in European TB reference centres: the Achilles's heel? Eur Respir J 2011; 38: 1221-1223.

6 Ling DI, Zwerling AA, Pai M. GenoType MTBDR assays for the diagnosis of multidrug-resistant tuberculosis: a meta-analysis. Eur Respir J 2008; 32: 1165-1174

7 World Health Organization. Policy Statement: Molecular Line Probe Assays For Rapid Screening of Patients at Risk of MultidrugResistant Tuberculosis. Geneva, World Health Organization, 2008. www.who.int/tb/laboratory/line_probe_assays/en/. Date last updated June 27, 2008. Date last accessed July 29, 2011.

8 Hillemann D, Rüsch-Gerdes S, Richter E. Feasibility of the GenoType MTBDRsl assay for fluoroquinolone, amikacin-capreomycin, and ethambutol resistance testing of Mycobacterium tuberculosis strains and clinical specimens. J Clin Microbiol 2009; 47: 1767-1772.

9 Brossier F, Veziris N, Aubry A, et al. Detection by GenoType MTBDRsl test of complex mechanisms of resistance to second-line drugs and ethambutol in multidrug-resistant Mycobacterium tuberculosis complex isolates. J Clin Microbiol 2010; 48: 1683-1689.

10 van Ingen J, Simons S, de Zwaan R, et al. Comparative study on genotypic and phenotypic second-line drug resistance testing of Mycobacterium tuberculosis complex isolates. J Clin Microbiol 2010; 48: 2749-2753.

11 Kiet VS, Lan NT, An DD, et al. Evaluation of the MTBDRsl test for detection of second-line-drug resistance in Mycobacterium tuberculosis. J Clin Microbiol 2010; 48: 2934-2939.

12 Huang WL, Chi TL, Wu MH, et al. Performance assessment of the GenoType MTBDRsl test and DNA sequencing for the detection of second-line and ethambutol drug resistance among patients with multidrug-resistant Mycobacterium tuberculosis. J Clin Microbiol 2011; 49: 2502-2508.

13 Gagneux S, Small PM. Global phylogeography of Mycobacterium tuberculosis and implications for tuberculosis product development. Lancet Infect Dis 2007; 7: 328-337.

14 Parwati I, van Crevel R, van Soolingen D. Possible underlying mechanisms for successful emergence of the Mycobacterium tuberculosis Beijing genotype strains. Lancet Infect Dis 2010; 10: 103-111.

15 World Health Organization. Policy Guidance on Drug-Susceptibility Testing (DST) of Second-Line Antituberculosis Drugs. WHO/ HTM/TB/2008.392. Geneva, World Health Organization, 2008. Available from: whqlibdoc.who.int/hq/2008/WHO_HTM_TB_ 2008.392_eng.pdf

16 Miotto P, Piana F, Penati V, et al. Use of genotype MTBDR assay for molecular detection of rifampin and isoniazid resistance in Mycobacterium tuberculosis clinical strains isolated in Italy. J Clin Microbiol 2006; 44: 2485-2491.

17 Weniger T, Krawczyk J, Supply P, et al. MIRU-VNTRplus: a web tool for polyphasic genotyping of Mycobacterium tuberculosis complex bacteria. Nucleic Acids Res 2010; 38: Suppl. 2, W326-W331.

18 Leonard B, Coronel J, Siedner M, et al. Inter- and intra-assay reproducibility of microplate Alamar blue assay results for isoniazid, rifampicin, ethambutol, streptomycin, ciprofloxacin, and capreomycin drug susceptibility testing of Mycobacterium tuberculosis. J Clin Microbiol 2008; 46: 3526-3529.

19 Bossuyt PM, Reitsma JB, Bruns DE, et al. Towards complete and accurate reporting of studies of diagnostic accuracy: the STARD initiative. Fam Pract 2004; 21: 4-10.

20 Dean AG, Sullivan KM, Soe MM. OpenEpi: Open Source Epidemiologic Statistics for Public Health, Version 2.3.1. www. OpenEpi.com Date last updated October 19, 2010. Date last accessed May 16, 2011.

21 Campbell PJ, Morlock GP, Sikes RD, et al. Molecular detection of mutations associated with first- and second-line drug resistance compared with conventional drug susceptibility testing of Mycobacterium tuberculosis. Antimicrob Agents Chemother 2011; 55: 2032-2041.

22 Von Groll A, Martin A, Jureen P, et al. Fluoroquinolone resistance in Mycobacterium tuberculosis and mutations in gyrA and gyrB. Antimicrob Agents Chemother 2009; 53: 4498-4500.

23 Via LE, Cho SN, Hwang S, et al. Polymorphisms associated with resistance and cross-resistance to aminoglycosides and capreomycin in Mycobacterium tuberculosis isolates from South Korean Patients with drug-resistant tuberculosis. J Clin Microbiol 2010; 48: 402-411. 
24 Maus CE, Plikaytis BB, Shinnick TM. Molecular analysis of cross resistance to capreomycin, kanamycin, amikacin, and viomycin in Mycobacterium tuberculosis. Antimicrob Agents Chemother 2005; 49: 3192-3197.

25 Plinke C, Cox HS, Kalon S, et al. Tuberculosis ethambutol resistance: concordance between phenotypic and genotypic test results. Tuberculosis 2009; 89: 448-452.

26 Van Deun A, Wright A, Zignol M, et al. Drug susceptibility testing proficiency in the network of supranational reference laboratories. Int J Tuberc Lung Dis 2011; 15: 116-124.

27 Scarparo C, Ricordi P, Ruggiero G, et al. Evaluation of the fully automated BACTEC MGIT 960 system for testing susceptibility of Mycobacterium tuberculosis to pyrazinamide, streptomycin, isoniazid, rifampin, and ethambutol and comparison with the radiometric BACTEC 460TB method. J Clin Microbiol 2004; 42: 1109-1114.

28 Comas I, Gagneux S. The past and future of tuberculosis research. PLoS Pathog 2009; 5: e1000600.

29 World Health Organization. Report of the Tenth Meeting WHO Strategic and Technical Advisory Group for Tuberculosis (STAGTB) 27-29 September 2010. WHO/HTM/TB/2010.18. Geneva, World Health Organization, 2010.

30 The Global Laboratory Initiative. A Roadmap for Ensuring Quality Tuberculosis Diagnostics Services within National Laboratory Strategic Plans. Geneva, World Health Organization, 2010..

31 Baquero F. Low-level antibacterial resistance: a gateway to clinical resistance. Drug Resist Updat 2001; 4: 93-105. 